\title{
Der Starrheitssatz und die Deltaeder
}

\author{
Bernulf Weißbach \\ Meinem Lehrer Rudolf Prescher gewidmet \\ Bernulf Weißbach studierte von 1955 bis 1961 Mathematik an der Universität Jena. \\ Danach war er an der jetzigen Otto-von-Guericke-Universität in Magdeburg tätig, seit \\ 1992 als Professor für Geometrie. Sein Interesse gilt insbesondere der Geometrie in \\ Räumen beliebiger Dimension - dem Borsukschen Problem und verwandten Fragen.
}

\section{1 Übersicht}

Ein bedeutsamer Satz der Geometrie wird in Nachschlagewerken und auch anderswo oft so gefaßt, daß eine falsche Aussage zustande kommt. Es handelt sich um jenen Satz, mit dem sich A. Cauchy in einer seiner ersten Arbeiten beschäftigte. In dem für Lehrer an Gymnasien sowie für Mathematiker in Industrie und Wirtschaft bestimmten Sammelband „Grundzüge der Mathematik“ [1] erhielt er die Fassung

„Zwei isomorphe konvexe Polyeder sind kongruent, wenn ihre entsprechenden Flächen kongruent sind".

Eine gleichwertige Fassung bietet die „Encyclopaedia of Mathematics“ [6], ja schon im Beitrag von E. Steinitz in der „Encyclopädie der Mathematischen Wissenschaften“ [5] findet sich eine ähnliche falsche Aussage. Es gilt nämlich

Satz 1 Es gibt isomorphe aber inkongruente konvexe Polyeder, deren einander entsprechende Flächen kongruent sind.

Sicher ist es erstrebenswert, einen Satz knapp und einprägsam zu fassen. Werden dabei jedoch wesentliche Prämissen unterdrückt, so können sich fehlerhafte Aussagen ergeben. In der Literatur finden sich recht häufig unzureichende Fassungen des Starrheitssatzes von A. Cauchy. Dies wird augenfällig, wenn man konvexe Polyeder konstruieren will, die nur durch untereinander kongruente Dreiecke mit vorgegebener Gestalt begrenzt werden. Man findet Paare derartiger Polyeder, die zwar isomorph, aber nicht kongruent sind. 
Auf diesen Sachverhalt stößt man ganz en passant, wenn man versucht, sich einen Überblick über die einfachsten Typen von Deltaedern zu verschaffen. Deltaeder soll jedes konvexe Polyeder genannt werden, das von irgendwelchen untereinander kongruenten Dreiecken begrenzt wird. Dieser treffende Name wurde von M. Cundy [4] geprägt. Er wird bisher nur für jene konvexen Polyeder verwendet, deren Rand aus regulären Dreiecken besteht. Solche Polyeder wurden wohl zuerst 1915 durch O. Rausenberger näher betrachtet [8]. Jahre später haben H. Freudenthal und B.L. van der Waerden auf sie aufmerksam gemacht [7]. Werden zwei dieser speziellen Deltaeder als äquivalent angesehen, wenn sie zueinander ähnlich sind, so ordnen sie sich in nur acht Klassen ein.

Das Problem, alle allgemeinen Deltaeder geeignet aufzulisten, ist keineswegs einfach. Nicht umsonst haben es H.T. Croft, K.J. Falconer und R.K. Guy in ihre Sammlung „Unsolved Problems in Geometry“ [3] aufgenommen. Es heißt dort (Problem B 22):

„For which triangles $T$ does there exist a convex polyhedron with all of its faces congruent to T...? How many congruent triangles can be used, and how should they be arranged?"

Die erste dieser beiden Fragen ist allerdings sehr leicht zu beantworten. Es gilt

Satz 2 Zu jedem Dreieck T gibt es ein konvexes Polyeder, dessen Facetten alle zu T kongruent sind.

Um beide Sätze zu gewinnen, genügt es jene Deltaeder zu betrachten, in deren Ecken nicht mehr als vier Kanten zusammentreffen. Im weiteren wird ein Überblick über diese Polyeder gegeben.

\section{Deltaeder mit höchstens vierzähligen Ecken}

Die Anzahl der Ecken, Kanten und Facetten eines Polyeders sollen, wie heute allgemein üblich, mit $f_{i}, i=0,1,2$, bezeichnet werden. Wird $f_{0 k}$ für die Anzahl der Ecken gesetzt, in denen $k$ Kanten bzw. Facetten zusammentreffen, so gilt

$$
f_{0}=f_{03}+f_{04}+f_{05}+\cdots, \quad 2 f_{1}=3 f_{03}+4 f_{04}+5 f_{05}+\cdots
$$

Für Deltaeder ist $2 f_{1}=3 f_{2}$, und Eulers Gleichung $f_{0}-f_{1}+f_{2}=2$ liefert für die Zahlen $f_{0 k}$ die Kopplung

$$
3 f_{03}+2 f_{04}+f_{05}-\left(f_{07}+2 f_{08}+3 f_{09}+\cdots\right)=12 .
$$

Wird $f_{0 k}=0$ für $k \geq 5$ gefordert, so bleibt $3 f_{03}+2 f_{04}=12 \mathrm{zu}$ erfüllen. Es gibt nur drei Lösungen $\left(f_{03}, f_{04}\right)$, nämlich $(4,0),(0,6)$ und $(2,3)$.

Für $\left(f_{03}, f_{04}\right)=(4,0)$ und $f_{0 k}=0$ für $k \geq 5$ wird $\left(f_{0}, f_{1}, f_{2}\right)=(4,6,4)$, es handelt sich um Tetraeder. Es ist wohlbekannt, daß es Tetraeder mit zu einem vorgegebenen Dreieck $T$ kongruenten Facetten, sogenannte Disphenoide, genau dann gibt, wenn dieses Dreieck nur spitze Winkel aufweist. Sind die Kanten von $T$ paarweise inkongruent, so erscheinen die zugehörigen Disphenoide in zwei „,chiral“ zu unterscheidenden Formen. Tetraeder 
einer Klasse können durch eine eigentliche, Tetraeder aus unterschiedlichen Klassen nur durch eine uneigentliche Isometrie aufeinander abgebildet werden. Ist $T$ gleichschenklig, so fallen beide Klassen zusammen.

Für $\left(f_{03}, f_{04}\right)=(0,6)$ und $f_{0 k}=0$ für $k \geq 5$ wird $\left(f_{0}, f_{1}, f_{2}\right)=(6,12,8)$; diese Deltaeder sind zu regulären Oktaedern isomorph.

Im Schlegel-Diagramm eines konvexen Polyeders - einer kreuzungsfreien Darstellung des durch seine Ecken und Kanten bestimmten Graphen - lassen sich die Längen seiner Kanten durch Farben kenntlich machen. Wenn es zu einem Dreieck $T$ mit paarweise inkongruenten Seiten ein Deltaeder $D$ gibt, dessen Facetten zu $T$ kongruent sind, so lassen sich die Kanten des Diagramms von $D$ derart mittels dreier Farben färben, daß jeder 3-Kreis alle drei Farben trägt. Das Schlegel-Diagramm des regulären Oktaeders gestattet in der Tat derartige Färbungen. Es gibt sogar, wie Fig. 1 zeigt, zwei ganz verschiedene Typen zulässiger Färbungen. Beim Typ I treten drei 4-Kreise auf, deren Kanten jeweils die gleiche Farbe besitzen. Beim Typ II ist nur ein uni gefärbter 4-Kreis vorhanden; seine Farbe kann gewechselt werden.
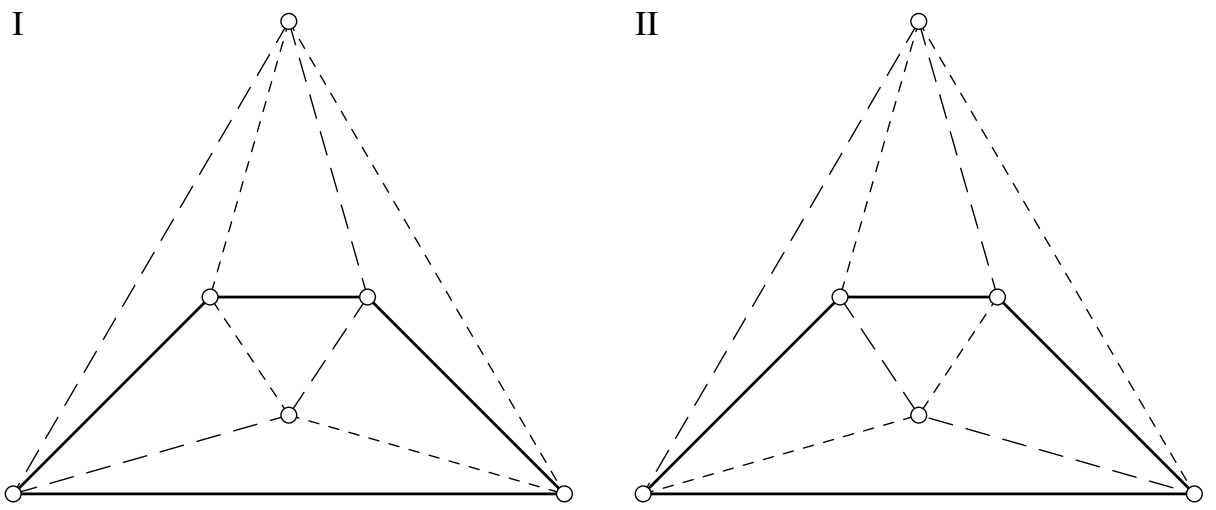

Fig. 1

Es muß gezeigt werden, daß es Deltaeder mit derart verhefteten Facetten gibt. Weiterhin sind die Dreiecke anzugeben, die in ihrem Rand auftreten können.

Aus den gefärbten Diagrammen kann die Gestalt zugehöriger Oktaeder voll erschlossen werden. Beim Typ I sind die den uni gefärbten 4-Kreisen entsprechenden Kantenzüge notwendigerweise eben, also Rhomben. Da je zwei dieser Rhomben eine gemeinsame Diagonale besitzen, ist ein zum Typ I gehörendes Oktaeder die konvexe Hülle dreier paarweise zueinander senkrechter Strecken $p_{i} \bar{p}_{i}, i=1,2,3$, deren Mittelpunkte zusammenfallen. Nur spitzwinklige Dreiecke können ein derartiges Oktaeder beranden; es kommt aber auch jedes spitzwinklige Dreieck $T$ dafür in Frage. Sind $l_{1}, l_{2}$ und $l_{3}$ die Längen der Seiten von $T$, so ist dieses Dreieck genau dann spitzwinklig, wenn die Bedingungen

$$
l_{i_{1}}^{2}<l_{i_{2}}^{2}+l_{i_{3}}^{2}, \quad\left\{i_{1}, i_{2}, i_{3}\right\}=\{1,2,3\},
$$


erfüllt sind. Dann gibt es aber positive Zahlen $k_{i}$ mit

$$
k_{i_{1}}^{2}=\frac{1}{2}\left(l_{i_{2}}^{2}+l_{i_{3}}^{2}-l_{i_{1}}^{2}\right), \quad l_{i_{1}}^{2}=k_{i_{2}}^{2}+k_{i_{3}}^{2},
$$

und $p_{1}\left(k_{1}, 0,0\right), p_{2}\left(0, k_{2}, 0\right), p_{3}\left(0,0, k_{3}\right)$ nebst $\bar{p}_{i}=-p_{i}$ sind die Ecken eines Oktaeders vom Typ I, dessen Facetten zu $T$ kongruent sind.

Beim Typ II betrachte man zwei Ecken des uni gefärbten 4-Kreises, die nicht durch eine Kante verbunden sind. Ersichtlich sind die restlichen vier Ecken von diesen beiden Punkten jeweils gleich weit entfernt. Diese vier Punkte liegen mithin in einer Ebene, sie sind die Ecken eines Drachenvierecks. Die beiden auftretenden Drachenvierecke sind kongruent, denn die Längen ihrer Seiten stimmen überein und sie besitzen eine gemeinsame Diagonale. Die beiden anderen Diagonalen dieser Drachenvierecke müssen sich senkrecht kreuzen, denn sie sind auch die Diagonalen eines von Strecken gleicher Länge gebildeten Vierecks. Folglich läßt sich auch ein zu diesem Typ gehörendes Oktaeder als die konvexe Hülle von drei Strecken $p_{i} \bar{p}_{i}$ mit paarweise zueinander senkrechten Richtungen ansehen. Zwei dieser Strecken sind gleich lang, ihre Mittelpunkte liegen auf der dritten Strecke. Deren Mittelpunkt halbiert die Strecke, die durch die Mittelpunkte der beiden anderen Strecken bestimmt wird (Fig. 2).
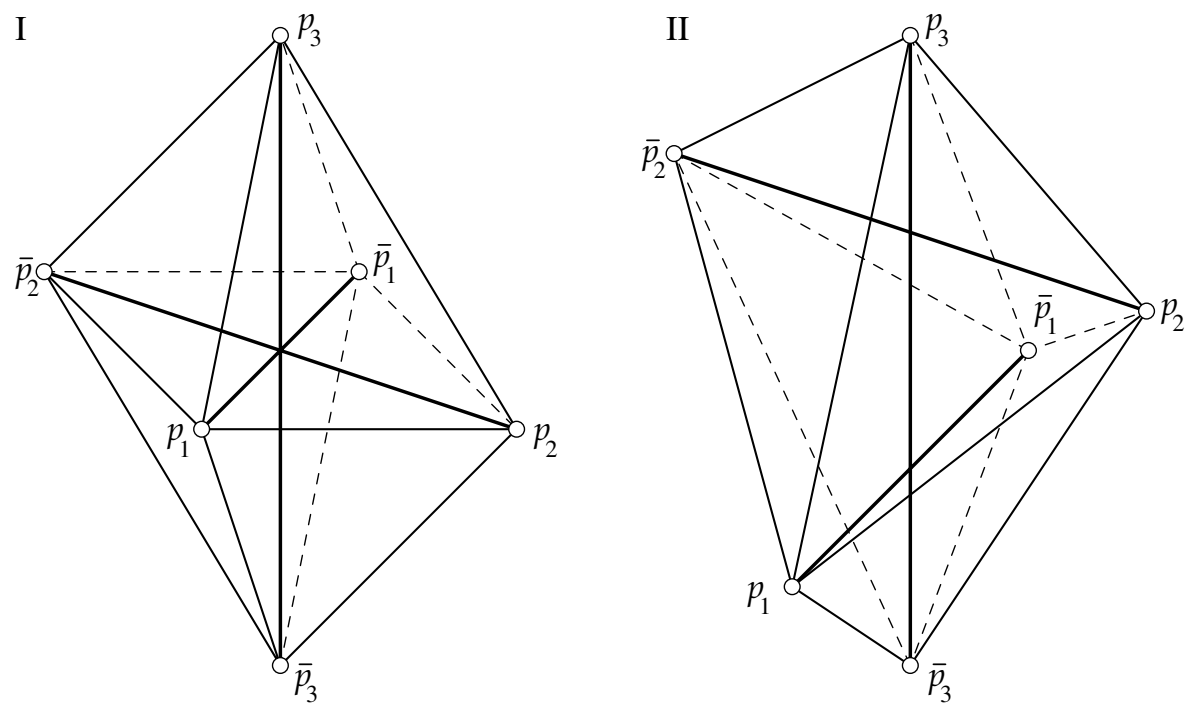

Fig. 2

Betrachtet man Oktaeder, die durch kongruente gleichschenklige Dreiecke begrenzt werden, so stößt man auf die gleichen Gestalten.

Beim Typ II soll die Bezeichnung der Ecken so vorgenommen werden, daß die Strecken $p_{1} \bar{p}_{1}$ und $p_{2} \bar{p}_{2}$ die gleiche Länge besitzen. Ursprung und Basis können dann so gewählt 
werden, daß die Ecken des Oktaeders durch

$$
\begin{array}{lll}
p_{1}\left(h_{0}, 0, h_{1}\right), & p_{2}\left(0, h_{0}, h_{2}\right), & p_{3}(0,0,0), \\
\bar{p}_{1}\left(-h_{0}, 0, h_{1}\right), & \bar{p}_{2}\left(0,-h_{0}, h_{2}\right), & \bar{p}_{3}\left(0,0, h_{1}+h_{2}\right)
\end{array}
$$

beschrieben werden, wobei die Zahlen $h_{i}$ positiv sind und $h_{1} \neq h_{2}$ gilt. Wird $h_{1}=h_{2}$ zugelassen, so werden gewisse Oktaeder des Typs I mit erfaßt. Dann lassen sich aber die Zahlen $h_{i}$ auch immer so bestimmen, daß das Dreieck mit diesen Ecken $p_{i} \mathrm{zu}$ einem beliebig vorgegebenen Dreieck $T$ kongruent ist. Dies gelingt, weil jedes Dreieck wenigstens zwei spitze Winkel besitzt. Angenommen im Dreieck $T$ schließen zwei Seiten mit den Längen $l_{1}$ und $l_{2}$ den spitzen Winkel mit der Größe $\alpha$ ein. Die Länge der dritten Seite von $T$ sei $l_{3}$, so daß $l_{3}^{2}=l_{1}^{2}+l_{2}^{2}-2 l_{1} l_{2} \cos \alpha$ gilt. Wegen $0<\alpha<\frac{\pi}{2}$ gelten für jede reelle Zahl $\varphi \in[0, \alpha]$ die Ungleichungen

$$
\cos \alpha \leq \frac{\cos \alpha}{\cos \varphi} \leq 1
$$

Es gibt dann eine Zahl $\bar{\varphi}$ aus dem Intervall $[0, \alpha]$, die stetig von $\varphi$ abhängt, und für die $\cos \varphi \cdot \cos \bar{\varphi}=\cos \alpha$ gilt. Insbesondere ist $\bar{\varphi}(0)=\alpha$ und $\bar{\varphi}(\alpha)=0$. Betrachtet man jetzt für einen zulässigen Wert von $\varphi$ die Punkte

$$
p_{1}\left(l_{2} \sin \varphi, 0, l_{2} \cos \varphi\right), \quad p_{2}\left(0, l_{1} \sin \bar{\varphi}, l_{1} \cos \bar{\varphi}\right), \quad p_{3}(0,0,0),
$$

so wird $\left\|p_{2}-p_{3}\right\|=l_{1},\left\|p_{1}-p_{3}\right\|=l_{2}$ und

$$
\left\|p_{1}-p_{2}\right\|^{2}=l_{1}^{2}+l_{2}^{2}-2 l_{1} l_{2} \cos \varphi \cos \bar{\varphi}=l_{1}^{2}+l_{2}^{2}-2 l_{1} l_{2} \cos \alpha=l_{3}^{2} .
$$

Man hat demnach ein zu T kongruentes Dreieck erhalten, dessen Ecken stetig von dem Parameter $\varphi$ abhängen. Die in $[0, \alpha]$ stetige Funktion $f(\varphi)=l_{2} \sin \varphi-l_{1} \sin \bar{\varphi}$ muß für einen Wert $\varphi_{0}$, dem $\bar{\varphi}_{0}$ zugewiesen ist, verschwinden, denn es ist $f(0)=-l_{1} \sin \alpha<0$ und $f(\alpha)=l_{2} \sin \alpha>0$. Nun kann

$$
l_{2} \sin \varphi_{0}=l_{1} \sin \bar{\varphi}_{0}=: h_{0}, \quad l_{2} \cos \varphi_{0}=: h_{1}, \quad l_{1} \cos \bar{\varphi}_{0}=: h_{2}
$$

gesetzt werden. Es ist noch anzumerken, daß $h_{1}=h_{2}$ auf $\tan \varphi_{0}=\tan \bar{\varphi}_{0}$ führt, woraus auf $\varphi_{0}=\bar{\varphi}_{0}$ und letztlich auf $l_{1}=l_{2}$ zu schließen ist.

Jedes Dreieck $T$, das nicht regulär ist, besitzt einen spitzen Winkel, an dem Seiten unterschiedlicher Länge anliegen. Wie soeben gezeigt, gibt es dann ein Oktaeder vom Typ II, dessen Facetten zu $T$ kongruent sind. Damit ist Satz 2 gewonnen. Besitzt $T$ drei verschiedene spitze Winkel, so kann man drei paarweise inkongruente Oktaeder vom Typ II mit zu $T$ kongruenten Facetten konstruieren. Außerdem gibt es dann noch das Oktaeder vom Typ I, welches zu den Oktaedern vom Typ II sicher nicht kongruent ist. Aber je zwei dieser vier Oktaeder sind isomorph - und die einander zugewiesenen Facetten sind gewiß kongruent. 
Ein noch einfacheres Beispiel, daß Satz 1 bestätigt, ergibt sich bei $\left(f_{03}, f_{04}\right)=(2,3)$. Die zugehörigen Deltaeder mit höchstens vierzähligen Ecken, für die $\left(f_{0}, f_{1}, f_{2}\right)=(5,9,6)$ gilt, sind Doppelpyramiden. Ist $T$ ein Dreieck mit paarweise inkongruenten Seiten, so gibt es abgesehen von Bewegungen höchstens eine derartige Doppelpyramide mit zu T kongruenten Facetten. Man erhält sie, indem man ein zu $T$ gehörendes Disphenoid $D$ an einer seiner Wände spiegelt, und das Spiegelbild $D^{*}$ mit $D$ vereinigt. Das Disphenoid $D$ existiert nur, wenn $T$ spitzwinklig ist. Diese Bedingung garantiert aber noch nicht, daß $D \cup D^{*}$ konvex ausfällt und von sechs Dreiecken begrenzt wird. Dies ist dann und nur dann der Fall, wenn sich die Höhen von $T$ in einem inneren Punkt jenes Dreiecks treffen, dessen Ecken die Mitten der Seiten von $T$ sind. Um diese Bedingung durch eine Forderung an die Längen $l_{i}$ der Seiten von $T$ zu erfassen, ist es zweckmäßig, wiederum das Dreieck $T$ mit den Ecken $p_{1}\left(k_{1}, 0,0\right), p_{2}\left(0, k_{2}, 0\right)$ und $p_{3}\left(0,0, k_{3}\right)$ zu betrachten, wobei $k_{i}>0$ durch $2 k_{i_{1}}^{2}=l_{i_{2}}^{2}+l_{i_{3}}^{2}-l_{i_{1}}^{2}$ bestimmt ist. Man zeigt nämlich leicht, daß bei diesem Dreieck der Schnittpunkt der Höhen genau dann im Innern des Dreiecks mit den Ecken $q_{1}\left(0, \frac{1}{2} k_{2}, \frac{1}{2} k_{3}\right), q_{2}\left(\frac{1}{2} k_{1}, 0, \frac{1}{2} k_{3}\right)$ und $q_{3}\left(\frac{1}{2} k_{1}, \frac{1}{2} k_{2}, 0\right)$ liegt, wenn die Ungleichungen

$$
\frac{1}{k_{i_{1}}^{2}}<\frac{1}{k_{i_{2}}^{2}}+\frac{1}{k_{i_{3}}^{2}}, \quad\left\{i_{1}, i_{2}, i_{3}\right\}=\{1,2,3\},
$$

erfüllt sind.

Ist $T$ gleichschenklig, und etwa $l_{1}=a, l_{2}=l_{3}=b$, so ergibt sich die Forderung $b>\frac{\sqrt{3}}{2} a$. Genau dann gibt es eine aus zwei Disphenoiden zusammengesetzte konvexe Doppelpyramide $P$, die von sechs zu $T$ kongruenten Dreiecken begrenzt wird. Es gibt aber dann eine zu $P$ inkongruente Doppelpyramide $\bar{P}$, für die letzteres ebenfalls zutrifft. Fig. 3 zeigt die beiden unterschiedlichen Färbungen des Schlegel-Diagramms. Doppelpyramiden des zweiten Typs gibt es schon für $b>\frac{\sqrt{3}}{3} a$.
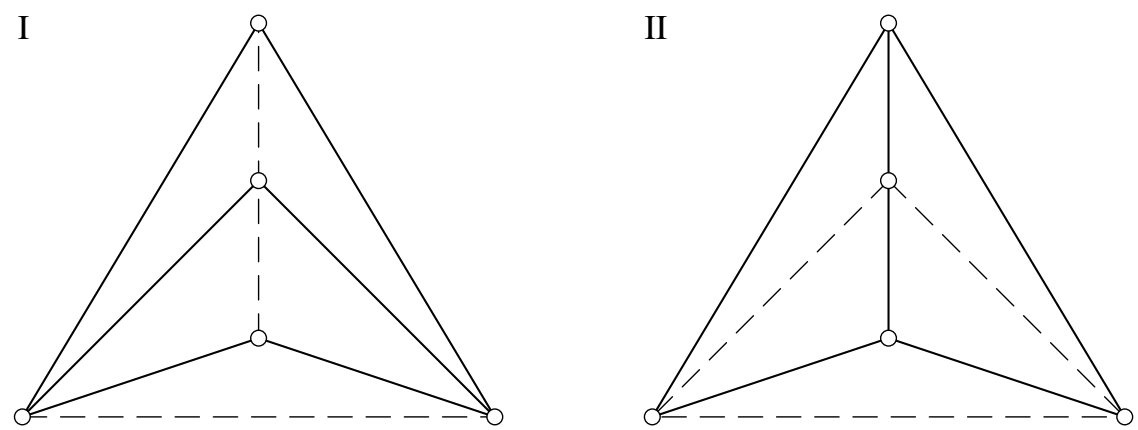

Fig. 3

$\mathrm{Zu}$ richtigen Fassungen des Starrheitssatzes gelangt man auf verschiedenen Wegen. Man kann breit ausholen; ein Beispiel bietet etwa M. Berger [2]. Es genügt aber, neben der Kongruenz der einander zugewiesenen Facetten auch die Kongruenz der einander entsprechenden Kanten zu fordern. Diese zweite notwendige Bedingung ist in den übrigen Forderungen eben noch nicht enthalten. 


\section{Literatur}

[1] Behnke, H.; Bachmann, F.; Fladt, K.; Süss, W. (Hrsg.): Grundzüge der Mathematik. Band II. Vandenhoeck \& Ruprecht, Göttingen 1960.

[2] Berger, M.: Geometry II. Springer Verlag, Berlin, Heidelberg 1987.

[3] Croft, H.T.; Falconer, K.J.; Guy, R.K.: Unsolved Problems in Geometry. Springer Verlag, New York 1991.

[4] Cundy, H.M.: Deltahedra. Math. Gazette 36 (1952), 263-266.

[5] Encyclopädie der Mathematischen Wissenschaften. B.G. Teubner, Leipzig 1914-1931.

[6] Encyclopaedia of Mathematics. Kluwer Academic Publishers, 1988.

[7] Freudenthal, H.; van der Waerden, B.L.: Over een Bewering van Euclides. Simon Stevin 25 (1947), 115-121.

[8] Rausenberger, O.: Konvexe pseudoreguläre Polyeder. Z. für den mathem. und naturwiss. Unterricht aller Schulgattungen 46 (1915), 135-142.

Bernulf Weißbach

Fakultät für Mathematik

Otto-von-Guericke-Universität Magdeburg

Postfach 4120

D-39016 Magdeburg, Deutschland 